\title{
Polémiques polémologiques
}

\section{Albert Galvany et Romain Graziani}

\section{(2) OpenEdition}

Journals

Édition électronique

URL : http://journals.openedition.org/extremeorient/348

DOI : 10.4000/extremeorient.348

ISSN : 2108-7105

Éditeur

Presses universitaires de Vincennes

\section{Édition imprimée}

Date de publication : 1 novembre 2014

Pagination : 5-20

ISBN : 978-2-84292-414-0

ISSN : 0754-5010

Référence électronique

Albert Galvany et Romain Graziani, «Polémiques polémologiques », Extrême-Orient Extrême-Occident

[En ligne], 38 | 2014, mis en ligne le 01 janvier 2015, consulté le 24 septembre 2020. URL : http://

journals.openedition.org/extremeorient/348; DOI : https://doi.org/10.4000/extremeorient.348 


\title{
Polémiques polémologiques
}

\author{
Albert Galvany et Romain Graziani
}

\section{Les nouvelles conquêtes de l'histoire militaire au $\mathrm{xx}^{\mathrm{e}}$ siècle}

Il paraît aujourd'hui légitime, voire justifié, d'entreprendre une enquête historique sur les déplacements massifs de populations civiles consécutifs à un conflit armé, d'analyser la participation des femmes aux préparatifs et à la conduite d'opérations militaires, de traiter de l'impact d'une action belliqueuse sur le fonctionnement des institutions ou de tout autre sujet touchant aux dimensions sociale, symbolique, culturelle, économique ou encore psychologique de la guerre. De telles orientations de l'esprit de connaissance sont pourtant le résultat d'une transformation récente, quoique profonde, dans notre manière de concevoir et d'écrire l'histoire. En Occident, les études consacrées à la guerre sont longtemps restées tributaires d'un intérêt prépondérant pour un nombre restreint de thèmes tels que la tactique, la logistique, la technologie ou encore l'armement utilisé au cours des campagnes militaires. Parallèlement à ces analyses, avaient le vent en poupe les biographies des grands généraux et stratèges dont l'histoire a fait des protagonistes de premier choix, liés de façon inextricable au destin de la nation ${ }^{1}$.

Au cours de la première moitié du $\mathrm{Xx}^{\mathrm{e}}$ siècle, ce type d'histoire traditionnelle se maintenait encore, certes comme une survivance obsolète - sinon suspecte à la marge des grands courants de pensée qui étaient en train de renouveler en profondeur l'historiographie, au premier chef l'école marxiste et celle des Annales. L'intérêt pour les batailles et les guerres commençait alors à être perçu, et cela de manière croissante et irrémédiable, comme le signe d'une mentalité antiquaire, pour être finalement relégué au rang de divertissement

1. Garlan (1972) : 7. Sur l'approche traditionnelle de l'étude du phénomène guerrier, voir, entre autres références, Morillo (2006) : 11-37. 
pour amateurs enthousiastes dépourvus de la plus élémentaire formation scientifique $^{2}$.

À la base de grands récits descriptifs, que maints historiens modernes imputent soit à la ferveur nationaliste soit à une inspiration exclusivement belliciste tout en en dénonçant le caractère répétitif et vide de sens, cette approche traditionnelle de la guerre exerça son influence jusqu'à la fin des années 1960. Encore au début des années 1970, l'historien Gordon A. Craig déplorait l'absence de recherches fiables et rigoureuses sur la guerre dans l'historiographie contemporaine ${ }^{3}$, et cela en dépit d'une amorce de changement dès cette époque. C'est à ce moment en effet qu'apparaissent les premiers indices d'une attitude qui, en quelques années, finira par opérer une révision radicale de l'historiographie traditionnelle et le rejet total des préjugés qui entachaient jusque-là les historiens de profession. Ici et là se détectent les signes précurseurs d'un renouveau des études sur la guerre et d'un élargissement des perspectives adoptées. Citons parmi d'autres l'exemple du livre de Georges Duby, Le Dimanche de Bouvines, publié en 1973, ou le cours que Michel Foucault dispense au Collège de France en 1975-1976, qui propose une analyse critique du rôle joué par la guerre et la pensée militaire dans la formation des États modernes, ou encore les deux volumes que, cette même année, Raymond Aron consacre à l'œuvre de Clausewitz ${ }^{4}$. À partir de 1980 , l'ostracisme académique dont étaient victimes les études sur la guerre cesse progressivement de s'exercer sous l'effet de perspectives, de méthodologies et d'enjeux inédits pour ouvrir la voie à ce que le monde anglo-saxon baptise la Nouvelle histoire militaire (New Military History) ${ }^{5}$. Précisant la portée de ce nouveau regard sur la guerre à l'occasion d'un discours devant la Society of Military History, le 23 mars 1991, Peter Paret en mentionne l'un des traits distinctifs : l'expansion de l'objet même de ce type d'histoire. Après s'être occupée des caractéristiques propres à l'organisation et à l'action militaires, cette discipline rénovée en est venue, en effet, à élargir son champ de vision pour traiter des implications sociales et culturelles des conflits armés ${ }^{6}$. Force

2. Fagan \& Trundle (2010) : 6. On retrouve le même rejet et la même mise à l'écart dans le domaine de l'archéologie. Voir à ce sujet les arguments avancés par Pollard \& Banks (2005) : iii-vii.

3. Craig (1971) : 323-348, cité par Loreto (2006) : 6 .

4. Duby (1973); Foucault (1997) : 3-36; Aron (1976).

5. Au sujet de cette expression et de son emploi, je renvoie le lecteur à l'ouvrage de Black (2004) : 49-58. Pour un débat sur l'apparition de la Nouvelle histoire militaire et l'exposé de ses objets d'étude et sa méthodologie, voir, entre autres, les contributions de Loreto (2006) et de Citino (2007) : 1070-1090.

6. Cité in Chambers II (1991) : 397. Voir aussi P. Paret (1991) : 10-18. 
est donc de constater que ce n'est qu'au cours des dernières décennies du $\mathrm{xx}^{\mathrm{e}}$ siècle que la science historique en Occident a cessé d'aborder la guerre exclusivement à partir des modèles traditionnels, ceux que désignent encore aujourd'hui les termes dépréciatifs de "tambours et clairons » (drums and trumpets). L'intégration de ce champ de recherches aux sciences historiques et l'adoption d'une multitude de perspectives sur le phénomène de la guerre sont, comme on le voit, une conquête des plus récentes. L'une des conséquences les plus sensibles de cette rupture épistémologique dans l'approche de la guerre, construite comme objet culturel, phénomène économique ou encore enjeu symbolique, fut de revitaliser l'histoire militaire en lui proposant de nouvelles pistes de questionnement, en diversifiant ses méthodes et en modernisant son appareillage optique si l'on peut dire. Or, une telle rupture s'est produite, étrangement, au moment même où la guerre, comprise comme conflit armé, public et inscrit dans un cadre juridique, semble dépérir, voire même entièrement disparaître ${ }^{7}$.

\section{D’un préjugé tenace sur la civilisation chinoise}

Bien que longtemps repliée sur des paradigmes datés et des méthodes anciennes, la sinologie s'est assez vite acclimatée à ces nouveaux courants historiographiques : signalons dès 1974 l'une des premières tentatives d'approche de la question de la guerre dans une perspective historique large avec la publication d'un volume collectif dirigé par Frank Kierman et John Fairbank, Des Voies de la guerre en Chine (China's ways in warfare) ${ }^{8}$. Les sinologues avaient devant eux un large chantier, étant donné la riche tradition de traités militaires et de réflexions tactiques et stratégiques, inaugurée dès l'aube $d u v^{e}$ siècle avant notre ère et dont la vitalité spéculative s'était maintenue depuis lors sans interruption ${ }^{9}$. Le renouvellement du champ d'investigation

7. Telle est, du moins, la conception de plusieurs penseurs et théoriciens contemporains de la politique qui considèrent que la guerre, dans sa forme classique, a aujourd'hui disparu au profit de la notion d' « intervention », plus proche des institutions sécuritaires ou policières. C'est la thèse défendue entre autres par Giorgio Agamben (2002).

8. Kierman \& Fairbank (1974). Il convient également de noter qu'un an auparavant, mais couvrant une période bien plus limitée, paraît l'ouvrage de William \& Chen-hsia Huang Whitson sur les affaires militaires à l'époque communiste (1973).

9. La quantité de textes militaires produits en Chine au cours des siècles est immense. Selon les estimations non exhaustives de Liu Shenning 劉申寧 (1990) de l'Antiquité jusqu'à la fin de la dynastie Qing (1911), on compterait quelque 4221 textes et traités (cité dans Yates [2005] : 66). 
que résume le nom de «guerre» fut pourtant ralenti, dans le cas présent, par un préjugé tenace, qui tient à l'image que la Chine s'est forgée de sa propre culture et de ses institutions, tout au moins à partir de la dynastie Song (9601279).

Selon l'interprétation avancée par Alastair Iain Johnston, il faudrait en effet distinguer, à partir du second millénaire, deux attitudes fondamentales face à la guerre et à la culture militaire : d'un côté, ce que l'historien canadien appelle le paradigme « confuciano-mencien », c'est-à-dire hérité directement de la pensée de Confucius puis de l'oeuvre écrite de son sectateur Mencius (380-289 av. J.-C) ; de l'autre, ce qu'il baptise le paradigme parabellum. Selon le premier, toute forme de violence ou de coercition doit être rejetée au profit d'une attitude morale de la part du gouvernement, supposément suffisante pour déjouer les menaces contre la sécurité intérieure, tout en ayant recours, s'agissant de sécurité extérieure, aux manœuvres diplomatiques, aux alliances, aux tributs et aux relations commerciales. Le paradigme parabellum, au contraire, intègre l'affrontement armé comme élément constant des rapports entre États et, par conséquent, accepte le principe de la violence légale comme moyen le plus efficace de gérer les conflits. Selon ce second paradigme, la sécurité intérieure, reposerait, autant que la sécurité extérieure, sur la force militaire, seule susceptible d'assurer la défaite, voire l'anéantissement, de toute ennemi ${ }^{10}$. Pour Johnston, comme pour d'autres auteurs ${ }^{11}$, l'opinion prévalant tant en Chine qu'en Occident ${ }^{12}$ tient la civilisation chinoise pour réfractaire à toute forme de militarisme et adhérant pleinement aux valeurs essentielles du confucianisme. Nimbé par l'aura de la figure tutélaire de Confucius, modelé sur la conduite du sage opposé à l'usage de la violence, travaillé par la doctrine impériale d'un Etat se voulant lui-même confucéen et prônant sans relâche l'étude des textes classiques contribuant à l'édification morale de chacun, le pacifisme l'aurait emporté sur toute forme de culture martiale dans l'histoire chinoise, et ce de manière croissante depuis le début du second millénaire. C'est ainsi que la sphère civile serait parvenue à s'imposer au domaine militaire

10. Voir Johnston (2005) : 61-108.

11. Voir, par exemple, ter Haar (2000) : 123-140.

12. On peut citer pour son caractère symptomatique l'attitude de Matteo Ricci qui, dans son œuvre, souligne l'influence quasi exclusive de l'héritage confucéen et n'hésite pas à décrire la civilisation chinoise comme fondamentalement pacifique, exempte de militarisme agressif. Voir à ce sujet, Mungello (1985) : 66-67. On retrouve dans l'œuvre de Max Weber [1915] cette identification de la civilisation chinoise au confucianisme et au pacifisme qui lui est attribué, ce qui ne fait que renforcer cette tendance à l'essentialisme. 
pendant une grande partie de l'histoire impériale chinoise ${ }^{13}$. Cette image pérenne se retrouve dans les textes et discours officiels des dirigeants chinois d'aujourd'hui - militaires ou non - où se rappelle constamment la prégnance de cette tradition pacifiste plongeant ses racines dans le terreau du confucianisme antique. Les exemples sont légion, mentionnons parmi tant d'autres Li Jijun, lieutenant-général de l'Académie des sciences militaires (Junshi kexueyuan), qui considère que la culture militaire chinoise est fondamentalement de caractère défensif. Ou encore l'ancien Premier ministre Wen Jiabao, lequel soulignait au cours d'une conférence donnée en 2003 à l'université Harvard le penchant de la nation chinoise pour la paix et le caractère défensif de sa politique internationale depuis des siècles ${ }^{14}$.

\section{Confucius bellicosus ?}

La fréquentation familière des textes anciens et leur recoupement critique permet toutefois de mesurer combien ce paradigme pacifiste confucianomencien proposé par l'historien Alastair Iain Johnston relève en vérité d'un mélange indécidable d'ignorance et de mauvaise foi. La conduite et la carrière de Confucius, si elles n'en font pas un stratège ou un partisan de la force armée, montrent néanmoins de façon indiscutable le caractère martial de ses décisions et la préférence nette qu'il pouvait accorder aux châtiments punitifs au détriment de l'éducation correctrice en certaines occasions.

Observons d'emblée que l'image répandue d'un Confucius explicitement opposé à toute forme d' agression ou de violence n'est fondée la plupart du temps que sur l'interprétation d'un nombre très modique de fragments provenant presque invariablement des Entretiens ${ }^{15}$. Dans de nombreux textes qui s'étendent de la période pré-impériale jusqu'aux Han, le portrait de Confucius et son rapport à la violence d'Etat s'avèrent beaucoup plus ambigus. On trouve par exemple dans le compendium des rites intitulé Da Dai Liji un passage qui traite de l'émergence de la violence et de l'invention des armes dans lequel Confucius justifie leur usage : « Le Duc demanda : "L'usage des armes procède-

13. Voir, par exemple, Fairbank (1974) : 7-9 ; Sawyer (1996) : 3 ; Mancall (1984) : 11 ; Adelman \& Shih, (1993) : 30-32; Bell (2008) : 35.

14. Li Jijun 李際均 (1997.1) : 8-15, et (2003) «Remarks of Chinese Premier Wen Jiabao : “Turning Your Eyes to China" ». Références citées par Wang (2011). Des thèses très proches sont rapportées également dans Gong Yuzhen 宮玉振 (2002) et Tiejun Zhang (2002) : 73-90.

15. Lunyu 論語 VII.21 ; XII.7 ; XIV.16 ; XVI.7 
t-il de l'infortune ?” À quoi le Maître répondit : “Comment pourrait-il procéder de l'infortune ? Les sages ont utilisé les armes pour proscrire la cruauté et mettre fin à l'oppression dans le monde entier ${ }^{16}$ ". » Dans un autre passage appartenant aux Propos de l'école de Confucius (Kongzi jiayu), un disciple de Confucius, Ran You (connu également sous le nom de Ziyou) s'apprête à offrir ses services au puissant lignage Ji dans le pays de Lu face à la menace imminente d'une agression, et démontrer ainsi son expertise en matière de stratégie militaire. Son supérieur, Jisun, étonné de voir qu'un disciple direct de Confucius ait pu cultiver de telles compétences lui demande comment, se réclamant du maître, il a bien pu étudier la stratégie militaire. À quoi Ran You répond : "Précisément, j'ai appris cela de Confucius. Confucius est un grand sage et, comme tel, rien n'est hors de la portée de son discernement ${ }^{17}$. »

Parmi les disciples les plus proches, on trouve également Zilu, un ancien guerrier de carrière à la nature belliqueuse et qui aimait à ferrailler ; son caractère hautain et intransigeant le prédisposait à des saillies de violence que son Maître avait bien de la peine à contenir ou modérer. Sa male mort scella ce destin guerrier : Le Mémoire sur les rites (Liji) rapporte en effet par la bouche d'un messager s'adressant à Confucius : «Avec sa chair ils ont fait du pâté au vinaigre ${ }^{18} »$. Citons encore cet autre disciple du nom de Gongliang $\mathrm{Ru}$, connu aussi sous celui de Zizheng, qui selon l'historien des Han Sima Qian disposait de cinq chars de guerre et alliait une extrême bravoure à une grande vigueur physique, qui lui servit à combattre, en compagnie du Maître, les gens de $\mathrm{Pu}$ au moment d'une révolte ${ }^{19}$. Confucius n'hésite pas pour sa part à faire l'éloge de personnages guerriers et de leurs faits militaires, comme lorsqu'il loue Meng Zhifan dans un passage des Entretiens (Lunyu VI.13). D'autres sources ${ }^{20}$ rapportent qu'il mena lui-même des opérations militaires, notamment lorsque les habitants de Bi attaquèrent la capitale de Lu en 497 avant notre ère.

La considération de ces passages comme de tant d'autres relatant des moments de la vie de Confucius laisse penser que ce dernier était loin d'être étranger au paradigme que Johnston baptise parabellum. Le pacifisme du Maître est en effet comme inquiété par d'autres épisodes de sa vie politique, qui montrent qu'il se rangeait sans réserve du côté de la méthode forte, aux

16. Wang Pinzhen 王聘珍 (1998) : 209.

17. Voir Kongzi jiayu shu zheng 孔子家語疏証. Édition établie par Chen Shike 陳士珂. Shanghai : Shanghai shudian (1980).

18. Couvreur (1950) : t. 1, 52. Voir aussi Zuozhuan, duc Ai, 15e année, in Yang Bojun 楊 伯峻 (1995) : 1694-1696.

19. Shiji, XLVII : 1923.

20. Zuozhuan, duc Ding, $12^{\mathrm{e}}$ année, in Yang Bojun 楊伯峻 (1995) : 1586-1587. 
antipodes du portrait du saint construit par l'idéologie impériale, opposé à la violence, prêchant la bonté et la justice. Mentionnons pour preuve, ce n'est pas le lieu ici de commenter en longueur ces épisodes, la fameuse entrevue diplomatique de Jiagu entre le duc de Lu, que servait Confucius, et le puissant duc de Qi. Après s'être senti menacé par une troupe de danseurs martiaux dans les gestes desquels il flairait un attentat possible et qu'il écarta en invoquant la correction rituelle, Confucius fit dépecer une troupe de nains ${ }^{21}$, danseuses et bouffons au motif qu'il était contraire aux rites de les faire parader sur scène. C'était là un fin coup de diplomate et de stratège pour faire plier le duc de Qi et obtenir des avantages matériels en se récriminant haut et fort sur l'entorse de ce dernier au protocole d'entrevue. Autant pour la bonté et la droiture légendaire du maître, ici éclipsées au profit d'une superbe leçon de cynisme politique. L'ascendant moral de Confucius allié à la mâle énergie qu'il manifeste et qui lui permet de faire déférer un puissant duc au nom des convenances, confirme la citation de son disciple Ran You reproduite plus haut : en lui convergent les valeurs martiales et militaires, le pinceau et le fouet, la natte et le char. Un second épisode achève le trait de ce portrait martial. Nommé aux fonctions de ministre dans l'Etat de $\mathrm{Lu}$, sa première décision fut de faire exécuter un dissident, du nom de Shao Zhengmao, au motif que ce dernier abusait de la crédulité du peuple par ses raisonnements spécieux et ne cessait de gagner en popularité22.

De tels événements, que corroborent les sources historiques et que les auteurs anciens mettent à l'honneur de Confucius, ne manquent pas d'invalider l'idée si répandue à propos de la Chine d'une tradition pacifiste, gouvernée par la doctrine confucéenne et imprégnée par l'esprit des lettres. Sous l'impulsion des confucéens tout autant que des légistes, et peu importent ici les désignations d'école, les méthodes martiales conçues dans le contexte d'une guerre intestine ou inter-étatique se sont introduites dans l'administration du corps politique sans solution de continuité et ont permis de renforcer l'arsenal déjà sophistiqué des règles et prohibitions qui gouvernaient les relations hiérarchiques. Au demeurant, Confucius et ses sectateurs, en dépit de quelques déclarations tirées des Entretiens hâtivement brandies pour « sauver » le pédagogue humaniste et prouver que leur seul recours était l'exemple moral, ne se sont en réalité jamais

21. L'historien dit : Shouzu yi chu 手足異處 : littéralement « les pieds et les mains en des endroits différents » une expression légale pour indiquer le fait de trancher à vif une victime en deux au niveau de la taille (yaozhan 腰斬).

22. Un épisode de la vie de Confucius rapporté notamment au chapitre 28 du Xunzi, « You zuo 宥左》. 
dans leurs actes ni leur carrière opposés aux châtiments qui établissent une continuité entre discipline martiale et éthique sociale.

Confucius bellicus ou Confucius bellicosus ? C'est en tout cas le Confucius historique lui-même que dans sa posture de chef martial viennent louer ses héritiers puis les historiens de l'ère impériale. Le premier des maîtres semble avoir fait sien ce précepte que devait énoncer haut et fort le penseur de l'autoritarisme politique Han Fei quelques trois siècles plus tard : « Entre un souverain et ses sujets, ce sont cent batailles par jour à livrer ». La politique est la gestion des guerres quotidiennes qui sévissent dans la société, contre les envieux, les vicieux et les séditieux, que l'on se situe sur le champ de bataille ou dans l'arène du politique

\section{En finir avec le mythe d'un « Art de la guerre » essentiellement chinois}

Ce volume s'attaque de manière frontale et massive à un autre préjugé qui a longtemps abusé les esprits à propos de la Chine. De nombreux sinologues ont bâti leurs succès de librairie, en Europe comme aux États-Unis, sur un brillant exposé des grandes différences qui opposent la civilisation occidentale à la Chine, en tirant de quelques idées fausses dès le départ un schéma général d'interprétation de leur développement. Alors que les Grecs de l'antiquité - dont nous sommes forcément les héritiers en ligne directe - auraient opté pour un combat frontal en bataille rangée, les Chinois auraient choisi la ruse, l'approche indirecte et le travail de sape de l'adversaire en amont de tout combat. La quintessence de quelques pages du livre de Sunzi, L'Art de la guerre - écrit au IV $\mathrm{IV}^{\mathrm{e}}$ siècle avant notre ère - recèlerait ainsi les clefs de la «mentalité chinoise ». Au risque d'occulter l'immense littérature stratégique et militaire contemporaine ou postérieure au Sunzi. Au risque aussi de confondre « guerre sur papier » (zhi shang tan bing) et histoire réelle des pratiques militaires en Chine. Or, la chronique des batailles, guerres et invasions qui ont marqué de façon si constante l'empire chinois pendant plus de deux millénaires, dément lourdement cette merveilleuse efficacité de la «stratégie indirecte » des Chinois.

La polarité Chine-Occident, construite à partir de deux attitudes stratégiques opposées dès l'antiquité, a parfois servi de modèle global pour caractériser deux façons typiques et opposées de penser et de s'exprimer : en Occident de façon argumentée, directe, chaque parole étant comme un brave hoplite bataillant contre la légion des arguments du camp adverse ; en Chine, de façon allusive, détournée - donc bien plus efficace - à la manière d'un général prenant 
l'ennemi à revers, ou en embuscade, sans se montrer, en évitant le corps-àcorps. Dans leurs travaux minutieux d'historiens des pratiques guerrières en Occident, Beatrice Heuser et Patrick Porter ${ }^{23}$ suggèrent que cette dualité de fond entre la civilisation chinoise et la civilisation occidentale reprend des thèses très anciennes, non dénuées de racisme, qui opposaient un Occident loyal, franc, honnête dans ses façons de combattre, à un Orient recourant à des expédients et des ruses moralement condamnables. L'extension de ce paradigme de la « stratégie à la chinoise » à la «pensée asiatique » en général se veut aujourd'hui, dans ses formes les plus sophistiquées, indemne bien sûr de ce préjugé raciste et neutre sur le plan des valeurs. Il n'en demeure pas moins qu'il conforte à son corps défendant le préjugé d'une mentalité chinoise encline à se montrer fourbe et sournoise. Le public mal informé des réalités de l'histoire chinoise en tire facilement la conclusion que les lettrés en Chine, qui étaient aussi les serviteurs du pouvoir impérial, ont toujours évité la polémique, le débat d'idées, la courageuse remontrance au souverain au risque de sa propre vie, au profit de formules ambiguës, savamment construites de manières à « passer » tout en suggérant de loin, et sans en avoir l'air, une critique ou un blâme. De même que le public, sous l'emprise de ce séduisant face-à-face entre la Chine et l'Occident qu'il s'imagine enfin déchiffrer, en vient à croire que les meilleurs généraux chinois ont su, contrairement à leurs homologues occidentaux, éviter le choc frontal et destructeur d'armées en campagne au profit de victoires obtenues sans avoir à livrer le combat. Ces lubies, tirées d'un texte antique aussi fascinant qu'idéaliste, et qui a longtemps servi de paravent occultant la vision lucide de ce que fut la guerre au quotidien dans l'empire chinois, - ces lubies ont joué un rôle prépondérant dans l'image contemporaine de la Chine, comme dans celle que, par différence, nous sommes amenés à construire de nous-mêmes. Si parmi les objectifs de ce numéro il s'agit de terrasser le préjugé d'une Chine confucéenne condamnant ou escamotant la violence militaire, les différents auteurs sont également unis par la volonté de creuser une sape pour faire s'écrouler la construction de cette grande fresque binaire retraçant d'un côté l'épopée de la civilisation occidentale, marquée par les guerres héroïques et les batailles décisives, de l'autre une Chine astucieuse et rusée qui machine et manigance pour gagner le combat en appliquant la méthode du non-agir.

Cette entreprise, dont il s'avère dès lors redondant de souligner le caractère engagé, est inaugurée par un article de Peter Lorge, intitulé « Discovering War in Chinese History », qui développe pour partie les idées que nous venons

23. Signalons par exemple l'ouvrage de Patrick Porter paru en 2009, Military Orientalism. Eastern War Through Western Eyes. 
d'introduire. Son texte se propose d'offrir au lecteur une réflexion de nature critique sur le rôle que la guerre et l'étude historique de cette dernière auraient joué en Chine selon la sinologie occidentale. Selon Lorge, les sinologues auraient accordé une attention bien plus grande à la pensée militaire qu'à l'histoire militaire. Dans le droit fil de cette argumentation, il retrace la genèse de ce qui semble avoir constitué un obstacle majeur à la juste compréhension du rôle joué par la guerre dans l'histoire chinoise : l'idée, étrangère à tout sens historique, selon laquelle il y aurait une manière essentiellement chinoise de conduire la guerre et les affaires militaire. En fonction de cette perspective essentialiste, l'attitude des intellectuels chinois a été rangée aux antipodes du bellicisme, tandis que, en parfait accord avec cette opinion, les stratèges et plus largement les penseurs en Chine qui se sont intéressés à la guerre auraient avant tout conçu et formulé une série de stratagèmes et de moyens contournés pour emporter la victoire. Lorge montre dans quelles conditions historiques et idéologiques le texte militaire traditionnellement attribué à Sunzi est devenu le paradigme de la pensée stratégique, et cela grâce sans doute à une lecture arbitraire qui a marginalisé la très riche et diverse tradition de commentaires de L'Art de la guerre, une mise à l'écart qui ne fit que se durcir sous l'effet d'une tendance prononcée à établir des comparaisons - dont on ne dira jamais assez le caractère biaisé - avec l'œuvre de Clausewitz. Incapables qu'elles sont de discerner correctement la dimension rhétorique des écrits militaires, Lorge considère que la plupart de ces interprétations influentes postulent de manière consciente ou non une parfaite continuité entre la littérature militaire et les pratiques guerrières, de sorte que les traités théoriques sur la guerre ne seraient que le parfait reflet de ces dernières, ce qui, comme on le pressent, constitue une hypothèse des plus problématiques. L'étude de Lorge montre à cet égard que l'instauration d'une distance critique par rapport à cette attitude essentialiste, comme par rapport aux notions qu'elle met en jeu, permettrait non seulement de revitaliser un champ d'études de première importance pour comprendre la Chine à travers l'histoire, mais aussi de reconsidérer l'intégralité de l'histoire militaire en Occident.

La contribution de David A. Graff, « Brain over Brawn : Shared Beliefs and Presumptions in Chinese and Western Strategemata », reprend et prolonge en quelque sorte les thèses avancées par Peter Lorge, en se proposant notamment d'invalider, à partir d'un exercice comparatif, un certain nombre d'idées reçues sur l'histoire militaire de la Chine. Comme on vient de le signaler, L'Art de la Guerre de Sunzi sert le plus souvent à caractériser la tradition militaire chinoise, dominée qu'elle serait par les manœuvres indirectes et l'emploi de la ruse, en contraste avec la manière occidentale de faire la guerre, incarnée dans l'œuvre de Clausewitz et définie par des attaques directes et frontales. Afin de saper 
les fondements de cette croyance aussi répandue que tenace, l'article de Graff prend pour objet d'étude les précis classiques de Frontin, Onasandre et Polyen $\left(\mathrm{I}^{\mathrm{er}}\right.$ et $\mathrm{II}^{\mathrm{e}}$ siècles de notre ère) ainsi que quelques traités militaires byzantins, en particulier celui attribué à l'empereur Maurice 1 ${ }^{\text {er }}$ (539-602), et, d'autre part, les écrits militaires chinois de l'antiquité, quelques histoires dynastiques ainsi que l'importante encyclopédie du viII ${ }^{\mathrm{e}}$ siècle intitulée Tong dian. En suivant le fil de son argumentation, on voit ainsi s'ébaucher différentes attitudes et notions relatives à l'emploi de la ruse, des artifices et des stratagèmes dans les cultures militaires gréco-byzantine et chinoise. À partir de cette perspective comparatiste, l'analyse de Graff insiste surtout sur la dimension psychologique des procédés stratégiques, en formulant les raisons pour lesquelles deux cultures aussi distinctes ont pu développer, au moins dans la littérature militaire qui subsiste aujourd'hui, aussi bien des modèles communs que des orientations divergentes.

Les deux contributions qui suivent, signées par Albert Galvany et Jean Levi, s'attaquent aux fondements idéologiques de la littérature militaire dans la Chine ancienne. Jean Levi part du constat que les discours et les institutions émanant de l'autorité politique définissent un arrangement particulier du droit et de la violence. À l'époque des Royaumes combattants s'échafaude une idéologie de la guerre juste qui excipe de notions morales pour légitimer des conflits jugés nécessaires. Jean Levi expose les conceptions divergentes de cette guerre juste (yi bing) et les modalités souvent irréconciliables de sa mise en œuvre en fonction des orientations des différents courants de pensée en Chine ancienne. À la lecture de son article, on a tôt fait de s'aviser que la guerre est le sujet polémique par excellence, que la notion de guerre juste est elle-même un champ de bataille dans lequel chaque penseur mobilise une légion d'arguments et affûte ses meilleurs lames pour imposer sa vision. Les stratèges et tacticiens qui mettent au point les formules de la victoire et sont les premiers à faire de la guerre un objet de pensée ou un champ de réflexion, ont tôt fait de se débarrasser des considérations rituelles et morales, senties comme autant de facteurs d'affaiblissement face à l'ennemi.

Abrités par le paravent moral qu'offre la réprobation bruyante et unanime de la guerre, les généraux et les stratèges sont, eux, à l'origine d'une littérature militaire qui célèbre le pouvoir destructeur du conflit armé et la dimension de drame cosmique en réduction que revêt le champ de bataille, tout en recommandant au capitaine d'armée avisé d'éviter tout choc sanglant et nuisible aux deux parties. L'exaltation des vertus belliqueuses chez le combattant est subordonnée à la considération d'un ordre rigoureux dans le déploiement de la force qui fait de chaque soldat non un héros potentiel en quête de renommée 
mais un élément dynamique ductile disposé dans un combat lui-même pensé comme une «partie en cours » et dont les combinaisons ont été anticipées.

Dans cette dernière perspective l'activité martiale, fut dans les textes comme dans le faits, strictement codifiée. A l'élan impétueux du soldat laissant éclater sa bravoure sur le champ de bataille se substitue un dispositif des corps auxquels ont été inculqués la syntaxe de gestes et de postures savamment répétés lors d'entraînements et d'exercices disciplinaires. C'est de cette dernière postulation qu'est née l'utopie régulatrice d'une guerre sans combat, de la victoire sans violence dont tant de sinologues ont fait leurs choux gras en occultant sciemment ou naïvement le fait qu'il s'agit là d'une idéologie entièrement coupée de la réalité historique et qui ne pouvait être formulée qu'au sein d'un univers de pensée surcodé, qui mettait en présence des ennemis nourris des mêmes références culturelles.

Loin d'accepter la tendance à comprendre les traités militaires chinois comme relevant d'une catégorie entièrement isolée des autres écoles ou doctrines de la Chine classique, l'article de Galvany se propose d'examiner la figure du général tel qu'elle est représentée dans diverses sources textuelles, qu'elles soient de nature politique, philosophique, historique ou proprement militaire. Dans cette littérature ancienne, le succès de toute action, en particulier des manœuvres militaires, est supposé dépendre du degré d'adéquation des acteurs à la conjoncture spatiale et temporelle toujours changeante ainsi que de leur capacité à prévoir les situations et à agir en conséquence. De ce point de vue, la figure du stratège, servie par une rhétorique compacte et puissante, est conçue dans ces textes à l'image des grandes figures de l'autorité morale et politique : dans sa sagesse infaillible et clairvoyante, le général des armées apparaît comme un individu capable d'anticiper les événements et de dévoiler la réalité interne des êtres grâce à la perception attentive des indices les plus ténus et leur juste interprétation. La pensée militaire en Chine ancienne attribue à cette figure idéalisée du stratège non seulement le talent de prévoir les événements dans le but de se projeter avec succès sur la chaîne du temps, mais encore la prudence de se tenir à l'abri de tout regard indiscret, de garder cachées ses intentions et de masquer ses plans en produisant des leurres et des fausses pistes afin d'intoxiquer l'adversaire. À partir de l'analyse d'épisodes militaires et de récits biographiques de quelques célèbres stratèges, le travail de Galvany montre les bénéfices et les risques liés à l'anticipation par interprétation d'indices et de traces.

Nonobstant l'emprise des préjugés pérennes et des distorsions idéologiques sur l'étude de la société et de l'histoire militaire chinoise, la sinologie est parvenue, au cours de ces dernières décennies, à se renouveler dans ses objets d'investigation et ses méthodes d'enquête. En parallèle au renouveau des 
études confucéennes qui au prix de raccourcis coupables louent l'aménité et la tolérance du premier des philosophes à l'égard de la gent humaine, l'histoire militaire chinoise connait aujourd'hui une progression notable avec la publication de nombreux travaux monographiques, d'ouvrages collectifs ainsi que d'une revue spécialisée ${ }^{24}$. On peut citer, entre autres, et par ordre chronologique de leur parution, les contributions de spécialistes tels que Mark E. Lewis, Raimund T. Kolb, Robin D. S. Yates, Hans van de Ven, Victoria T. Hui, Nicola Di Cosmo, David Graff, Peter Lorge, Ulrich Theobald ou Kenneth Swope $^{25}$. Le présent numéro, qui s'inscrit dans la vague de ce changement, se propose d'offrir au lecteur un recueil d'études, d'enquêtes et de réflexions traitant de la guerre à partir de perspectives temporelles ou géographiques fort diverses, mais toutes animées du souci de confirmer cette mue lente et profonde des études militaires, et de contribuer à la reconstruction concomitante de la Guerre comme objet de pensée, d'expérience et de savoir.

\section{BibliograPhiE}

Adelman Jonathan R. \& Shit Chih-yu (1993). Symbolic War : The Chinese Use of Force, 1840-1980. Taibei, Institute of International Relations.

Agamben Giorgio (2002). Moyens sans fins. Notes sur la politique. Paris, Rivages.

Aron Raymond (1976). Penser la guerre, Clausewitz.Paris, Gallimard.

Bell Daniel A. (2008). China's New Confucianism : Politics and Everyday Life in a Changing Society. Princeton, NJ, Princeton University Press.

BLACK Jeremy (2004). Rethinking Military History.London \& New York, Routledge.

Chambers II, J. W. (1991). « The New Military History : Myth and Reality ». Journal of Military History, $55.3: 397$.

CHEN Shike 陳士珂 (1980). Kongzi jiayu shu zheng 孔子家語疏証. Shanghai, Shanghai shudian.

Citino Robert M. (2007). « Military Histories Past an Present : A Reintroduction ». American Historical Review, 112.4 : 1070-1090.

Couvreur Séraphin (traducteur) (rééd. 1950). Mémoire sur les bienséances et les cérémonies. Paris, Cathasia.

24. La revue Journal of Chinese Military History, publiée par les éditions Brill et dirigée par David Graff et David C. Wright à partir de 2012, se propose de renouveler l'histoire militaire chinoise à partir de perspectives et de méthodologies innovantes initiées par la « Nouvelle histoire militaire».

25. Lewis (1990) ; Kolb (1991) ; Yates (1994) ; van de Ven (2000) ; Graff (2002) ; Graff \& Higham (2002) ; Lorge (2005a) et (2005b) ; Hui (2005) ; Di Cosmo (2013) ; Swope (2014). 
CRaIG Gordon A. (1971). « Political History ». Daedalus, 100.2 : 323-348.

DiCosmo Nicola (dir.) (2009). Military Culture in Imperial China. Cambridge, MA, Harvard University Press.

DuBy Georges (1973). Le Dimanche de Bouvines. Paris, Gallimard.

Fagan Garrett G. \& Trundle M. (dir.) (2010). New Perspectives on Ancient Warfare. Leyde, Brill.

Foucault Michel (1997). Il faut défendre la société. Cours au Collège de France, 1976.

Paris, Gallimard/Éditions du Seuil.

Garlan Yvon (1972). La Guerre dans l'Antiquité. Paris, Éditions Fernand Nathan.

GoNG Yuzhen 宮玉振 (2002). Zhongguo zhanlue wenhua jiexi 中國戰略文化解析.

Beijing, Junshi kexue chubanshe.

Graff David A. (2002). Medieval Chinese Warfare. New York, Routledge.

Graff David A. \& Higham Robin (2002). A Military History of China. Boulder, CO : Westview Press.

HAAR Barend ter (2000). «Rethinking "violence" in Chinese culture ». In G. Aijmer \& J. Abbink (dir.), Meanings of Violence : a Cross-Cultural Perspective.Oxford, Berg.

Hur Victoria T. (2005). War and State Formation in Ancient China and Modern Europe. Cambridge, Cambridge University Press.

Johnston Alastair Iain (2005). Cultural Realism : Strategic Culture and Grand Strategy in Chinese History. Princeton, NJ, Princeton University Press.

Kierman FranckA.\& Fairbank John F. (dir.) (1974). China's ways in warfare .Cambridge, MA, Harvard University Press.

KolB Raimund Theodor (1991). Die Infanterie im altem China : Ein Beitrag zur Militärgeschichte der Vor-Zhan-Guo-Zeit. Mainz am Rhein, von Zabern.

LEwIS Mark Edward (1990). Sanctioned Violence in Early China. Albany, NY, State University of New York Press.

Li Jijun 李際均 (1997). 《Lun zhanlue wenhua 論戰略文化》, Zhongguo junshi kexue, $1: 1-10$.

Li Jijun 李際均 (2003). « Remarks of Chinese Premier Wen Jiabao : 'Turning Your Eyes to China'». Talk at Harvard University, décembre 10.

LiU Shenning 劉申寧 (1990). Zongghuo bingshu zongmu 中國兵書總目. Beijing, Guofang daxue chubanshe.

LoReto Luigi (2006). Per la storia militare del mondo antico. Prospettive retrospettive. Naples, Jovene Editore.

LoRge Peter (2005a). War, Politics and Society in Early Modern China 900-1795. New York, Routledge.

Lorge Peter (2005b). Warfare in China to 1600. Burlington, VT, Ashgate.

Mancall Mark (1984). China at the Center : 900 Years of Foreign Policy. New York,

Free Press.

Morillo Stephen (2006). What is Military History ?.Cambridge, Polity Press.

Mungello David E. (1985). Curious Land : Jesuit accomodation and the origins of Sinology.Honolulu, University of Hawaii Press.

PARET Peter (1991). « The New Military History ». Parameters, 10 : 10-18. 
POLlaRd Tony \& BANKS Iain (2005). « Why a journal of conflict Archaeology and Why now ? ». Journal of conflict Archaeology, 1 : iii-vii.

Porter Patrick (2009). Military Orientalism. Eastern War Through Western Eyes. Londres, Hurst \& Company.

SAWYER Ralph D. (1996). The Art of the Warrior : Leadership and Strategy from the Chinese Military Classics. Boston, Shambhala.

Sima Qian 司馬遷 (1959). Shiji 史記.Beijing, Zhonghua shuju.

Swope Kenneth M. (2014). The Military Collapse of China's Ming Dynasty, 1618-1644. London, Routledge.

van de Ven Hans (dir.) (2000). Warfare in Chinese History. Leyde, Brill.

Wang Pinzhen 王聘珍 (1998). Da Dai Liji 大戴禮記. Beijing, Zhonghua shuju.

WANG Yuan-Kang (2011). Harmony and War : Confucian Culture and Chinese Power Politics. New York, Columbia University Press.

Weber Max ([1915] 1986). Konfuzianismus und Taoismus, in Gesammelte Aufsätze zur Religionssoziologie. Tübingen, J. C. B. Mohr.

Whitson William W. \& Whitson Chen-hsia (dir.) (1973). The Chinese High Command : A History of Communist Military Politics, 1927-1971. New York, Praeger Publishers.

YANG Bojun 楊伯峻 (1995). Chunqiu Zuozhuan zhu 春秋左傳注. Beijing, Zhonghua shuju.

Yates Robin D. S. (dir.) (1994). Science and Civilisation in China, vol. 5, Military Technology : Missiles and Sieges. Cambridge, Cambridge University Press.

YATES Robin D. S. (2005). « Early Modes on Interpretation of the Military Canons : The Case of the Sunzi bingfa ». In Ching-I Tu (dir.), Interpretation and Intellectual Change. Chinese Hermeneutics in Historical Perspective.New Brunswick, Transaction Publishers.

Zhang Tiejun (2002). « Chinese Strategic Culture : Traditional and Present Features ». Comparative Strategy, $21.2: 73-90$.

\section{Glossaire}

Da Dai Liji 大戴禮記

Gongliang $\mathrm{Ru}$ 公良孺

Jisun 季孫

Junshi kexueyuan 軍事科學院

Jiagu 夾谷

Kongzi jiayu 孔子家語

Li Jijun 李際均

Ran You 由有

Tongdian 通典

Wen Jiabao 溫家寶 
Albert Galvany et Romain Graziani

Zilu 子路

Ziyou 子游

Zizheng 子正 